\title{
Shift-Induced Dynamical Systems on Partitions and Compositions
}

\author{
Brian Hopkins \\ Department of Mathematics \\ Saint Peter's College, Jersey City, NJ 07306, USA \\ bhopkins@spc.edu \\ Michael A. Jones \\ Department of Mathematical Sciences \\ Montclair State University, Montclair, NJ 07043 \\ jonesm@mail.montclair.edu
}

Submitted: Feb 22, 2006; Accepted: Sep 10, 2006; Published: Sep 22, 2006

Mathematics Subject Classification: 05A17, 37E15

\begin{abstract}
The rules of "Bulgarian solitaire" are considered as an operation on the set of partitions to induce a finite dynamical system. We focus on partitions with no preimage under this operation, known as Garden of Eden points, and their relation to the partitions that are in cycles. These are the partitions of interest, as we show that starting from the Garden of Eden points leads through the entire dynamical system to all cycle partitions. A primary result concerns the number of Garden of Eden partitions (the number of cycle partitions is known from Brandt). The same operation and questions can be put in the context of compositions (ordered partitions), where we give stronger results.
\end{abstract}

\section{Introduction}

Let $P(n)$ be the set of partitions of $n$. The relation $\lambda \in P(n)$ will be written $\lambda \vdash n$. The shift operator $D_{P}: P(n) \rightarrow P(n)$ is defined as follows. Given a partition $\lambda=$ $\left(\lambda_{1}, \ldots, \lambda_{k}\right) \vdash n$, let $D_{P}(\lambda)$ be the partition of $n$ with parts $k, \lambda_{1}-1, \ldots, \lambda_{k}-1$, excluding any zeros (notice that the parts may not be in the standard nonincreasing order). The map is more easily defined from the graphic representation of a partition known as a Ferrers diagram: the first column of the diagram becomes a row, with reordering as needed to write the image in nonincreasing order. See Fig. 1. 


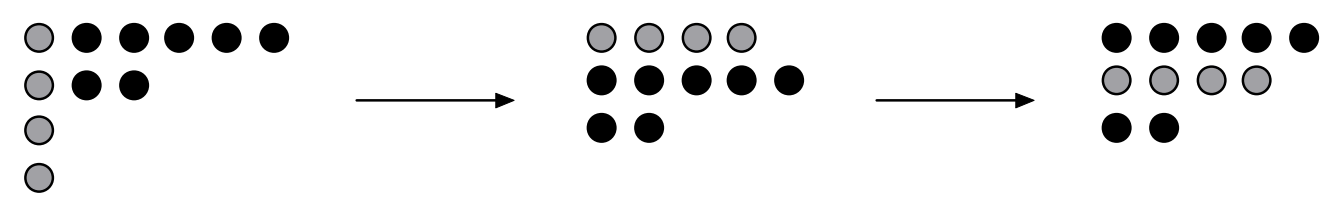

Figure 1: An example of the map on partitions: $D_{P}((6,3,1,1))=(5,4,2)$.

Analysis of this shift operator on partitions was first published by Jørgen Brandt in 1982 [4], although the author claimed that the problem had already "been circulating for some time." The next year, the idea was brought to a wider audience under the name "Bulgarian solitaire" in Martin Gardner's popular column [8]. A handful of papers followed contributing to the analysis of this operator, and several variants were introduced.

We will also consider the natural analog of the shift operation on $C(n)$, the set of compositions of $n$ (ordered partitions). The relation $\lambda \in C(n)$ will be written $\lambda \models$ $n$. Given a composition $\lambda=\left(\lambda_{1}, \ldots, \lambda_{k}\right) \models n$, let $D_{C}(\lambda)$ be the composition $\left(k, \lambda_{1}-\right.$ $\left.1, \ldots, \lambda_{k}-1\right)$ excluding any zeros. Thus $D_{C}$ is $D_{P}$ without reordering, but with the provision of closing gaps where there are now zeros. See Fig. 2.

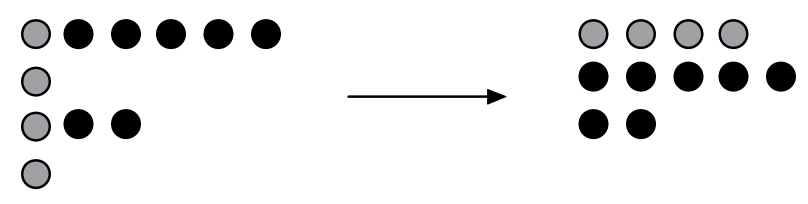

Figure 2: An example of the map on compositions: $D_{C}((6,1,3,1))=(4,5,2)$.

Here is some notation that we will use. Repeated elements are sometimes indicated by exponents, such as $\left(2,1^{4}\right)$ rather than $(2,1,1,1,1)$. In figures, we shorten the partition notation by representing $\left(2,1^{4}\right)$ as $21^{4}$. A partition or composition $\lambda$ with $k$ parts is said to have length $k$, written here $\ell(\lambda)=k$. Repeated application of the $D_{P}$ or $D_{C}$ map is denoted with exponents, e.g.,

$$
D_{C}^{2}((6,1,3,1))=D_{C}\left(D_{C}((6,1,3,1))\right)=D_{C}((4,5,2))=(3,3,4,1) .
$$

Recall the notion of the conjugate of a partition $\lambda$, written $\lambda^{\prime}$, which is most easily described in terms of the Ferrers diagram: reflect the dots across the diagonal, so that rows and columns switch roles. Some partitions are self-conjugate, while the rest fall into conjugate pairs. See Fig. 3.

Also, let $(k, \searrow, 1)$ indicate the list of integers decreasing by 1 . For $k=4,(k, \searrow, 1)$ is shorthand for $(4,3,2,1)$. We list only the greatest and least elements of such sublists. Lists with this notation sometimes collapse for small variable values. For example, when $k=3$, the abbreviated list in $(k+2, k-1, \searrow, 3,1)$ should be omitted entirely: the intended list is $(5,1)$. When $k=4$, the same notation denotes $(6,3,1)$; when $k=5$, it is $(7,4,3,1)$, etc. 


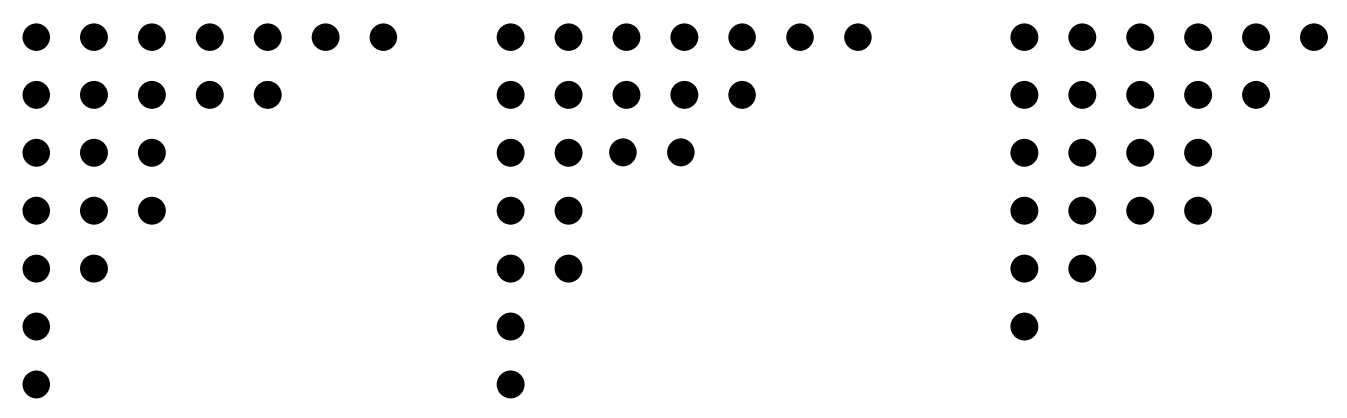

Figure 3: Conjugate partitions $(7,5,3,3,2,1,1),(7,5,4,2,2,1,1)$, and self-conjugate partition $(6,5,4,4,2,1)$, all in $P(22)$.

The cellular automata / finite dynamical systems context comes from considering the partition "state diagram," a directed graph whose vertices are elements of $P(n)$ and whose directed edges are the set of all $\left(\lambda, D_{P}(\lambda)\right)$. We can now define the objects of interest and outline of the paper.

Definition 1. A partition $\lambda$ is a cycle partition if $D_{P}^{m}(\lambda)=\lambda$ for some $m \geq 1$.

Cycle partitions are studied in [4] (details in Section 2). It is important to realize that $P(n)$ can contain multiple cycles, one per connected component of the corresponding directed graph.

Definition 2. A partition $\lambda$ is a Garden of Eden partition or GE-partition if $\lambda$ has no preimage under $D_{P}$.

The terminology comes from [12], a foundational paper in cellular automata. We denote by $G E_{P}(n)$ the set of GE-partitions in $P(n)$.

Definition 3. Given a GE-partition $\lambda$, its preperiod length is the least $m$ for which $D_{P}^{m}(\lambda)$ is a cycle partition.

In Section 2, we establish the importance of GE-partitions by proving that they are the entry points for all of $P(n)$ (for $n \geq 3$ ). The specific statement of Theorem 1 is that there are no stand-alone cycles, so that all cycles can be reached by starting at GE-partitions. Therefore, a complete analysis of $P(n)$ can be achieved by determining the GE-partitions and applying $D_{P}$ repeatedly to them. The proof of Theorem 1 establishes a stronger result, giving the minimal period length among the GE-partitions of $n$. This contributes to the program of understanding the complete distribution of GE-partition preperiod lengths, a primary part of studying the global dynamics of a system [15]. Previously, maximal preperiod lengths had been studied, along with selected intermediate values for particular $n$. Complete data for preperiod lengths in $P(n)$ up to $n=15$ are given in Section 4. The second primary result of Section 2 is a combinatorial proof establishing a lower bound for the size of $G E_{P}(n)$. 
All definitions apply to compositions, using the map $D_{C}$. In Section 3 , we consider analogous issues for GE-compositions. Again, we prove that there are no stand-alone cycles. The size of $G E_{C}(n)$ is computed exactly in two ways, giving a combinatorial proof of a Fibonacci number identity. In section 4, we will discuss various outstanding questions raised by our work.

\section{Partitions}

First, we summarize existing research relevant to our results. The initial observation on "Bulgarian solitaire" was that, for $n$ a triangular number $T_{s}=1+\cdots+s=s(s+$ 1) $/ 2$, repeated application of $D_{P}$ always leads to a single partition $\lambda=(s, \searrow, 1)$, which is fixed under $D_{P}$. For other $n$, repeated application of $D_{P}$ always leads to multiple cycle partitions. In addition to the results mentioned so far, [4] also gives a formula for determining the number of cycles for $n$, i.e., the number of connected components of the corresponding directed graph. The smallest state diagram with multiple components occurs at $n=8$ and is shown in Fig. 4 .

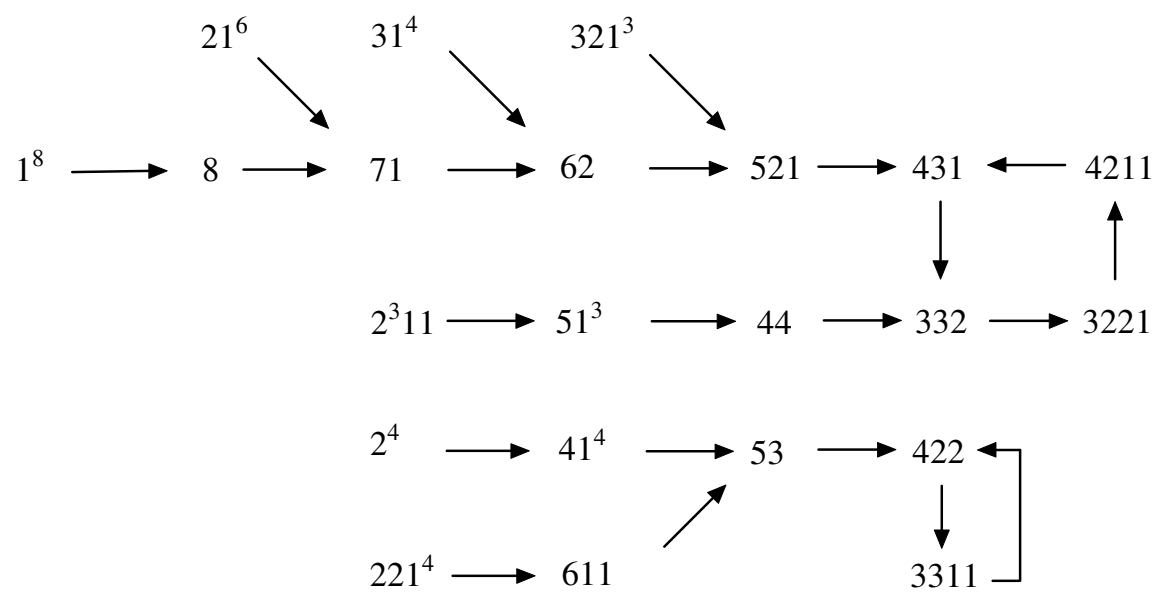

Figure 4: The two-component directed graph representing the state diagram for $D_{P}$ on $P(8)$.

We give the characterization of cycle partitions without proof, following the notation of [1], a helpful elaboration on [4]. For $n=T_{k}+r$ with $0 \leq r \leq k$,

$$
\lambda \vdash n \text { is a cycle partition } \Longleftrightarrow \lambda=\left(k+\delta_{k}, \ldots, 1+\delta_{1}, \delta_{0}\right)
$$

where exactly $r$ of the $\delta_{i}$ are 1 and the rest are 0 . Notice that cycle partitions have length $k$ or $k+1$, depending on $\delta_{0}$. We can completely describe cyclic $\lambda$ by $\Delta(\lambda)=\left(\delta_{k}, \ldots, \delta_{0}\right)$, a binary vector of length $k+1$. The effect of $D_{P}$ on cyclic $\lambda$ is cycling the entries of the vector $\Delta(\lambda)$. In particular,

$$
D_{P}(\lambda)=\left(k+\delta_{0}, k-1+\delta_{k}, \ldots, 1+\delta_{2}, \delta_{1}\right)
$$


and $\Delta\left(D_{P}(\lambda)\right)=\left(\delta_{0}, \delta_{k}, \ldots, \delta_{1}\right)$. For example, in the smaller component for $n=8$ shown in Fig. $4, \Delta((4,2,2))=(1,0,1,0)$ and $\Delta((3,3,1,1))=(0,1,0,1)$.

GE-partitions are characterized as a corollary to the following lemma, stated in [6] without proof.

Lemma 1. The number of $D_{p}$-preimages of a partition $\lambda$ is equal to the number of distinct parts $\lambda_{i} \geq \ell(\lambda)-1$.

Proof. Let $\lambda=\left(\lambda_{1}, \ldots, \lambda_{k}\right)$. For each distinct part $\lambda_{i} \geq k-1$, there exists a partition that maps to $\lambda$ under $D_{P}$. Specifically,

$$
D_{P}\left(\left(\lambda_{1}+1, \ldots, \lambda_{i-1}+1, \lambda_{i+1}+1, \ldots, \lambda_{k}+1,1^{\lambda_{i}-(k-1)}\right)\right)=\lambda \text {. }
$$

This implies that $\lambda$ has at least the number of $D_{P}$-preimages as the number of distinct parts $\lambda_{i} \geq \ell(\lambda)-1$.

Suppose $\kappa=\left(\kappa_{1}, \ldots, \kappa_{j}\right)$ and $D_{P}(\kappa)=\lambda . D_{P}(\kappa)$ consists of the nonzero parts in the unordered list $\kappa_{1}-1, \kappa_{2}-1, \ldots, \kappa_{j}-1, j$. Because $\ell(\lambda)=k$, there are $j+1-k$ zeros in the unordered list of $D_{P}(\kappa)$ 's parts. This implies that $\kappa_{i}=1$ for $i=k-1$ to $j$ so that $j \geq k-1$. Further, because $\ell(\kappa)=j$ must be a part in $D_{P}(\kappa)=\lambda$, then $j=\lambda_{i}$ for some $\lambda_{i} \geq k-1$. Therefore, $\kappa_{i}=\lambda_{i}+1$ for $i=1$ to $i-1$ and $\kappa_{i}=\lambda_{i+1}+1$ for $i=i$ to $k-1$. There exists a unique $D_{P}$-preimage for each distinct part $\lambda_{i} \geq \ell(\lambda)-1$.

Corollary 1. A partition $\lambda$ is a Garden of Eden partition if and only if $\ell(\lambda)>\lambda_{1}+1$.

The following theorem establishes the importance of studying GE-partitions in order to understand the $P(n)$ dynamical system determined by $D_{P}$. We prove that there are no stand-alone cycles, so that every cycle partition can be reached by starting from GEpartitions.

Theorem 1. For $n \geq 3$, every cycle partition $\lambda \in P(n)$ satisfies $D_{P}^{m}(\kappa)=\lambda$ for some $\kappa \in G E_{P}(n)$ and $m \geq 2$.

Proof. We show that every cycle partition has strictly positive minimal preperiod length. In fact, we show that, for $n=T_{k}$ with $k \geq 3$, the minimal preperiod length is 3 , and $n \neq T_{k}$ or $n=3$, the minimal preperiod length is 2 . The proof includes six cases. The initial comments and initial five cases prove the theorem - case 1 addresses the case where $n$ is a triangular number, and cases two through five cover cycle partitions for other $n$ in every connected component of the $P(n)$ directed graph determined by $D_{P}$. Case six establishes that there is GE-partition in some component of $n \neq T_{k}$ with minimal preperiod length 2.

First we show that there is no GE-partition with preperiod length 1 . Write $n=T_{k}+r$ where $0 \leq r \leq k$. We know that each cycle partition $\lambda$ satisfies $\ell(\lambda)=k$ or $k+1$. Since application of $D_{P}$ can increase partition length by at most 1 , any $\kappa \vdash n$ with $D_{P}(\kappa)=\lambda$ has $\ell(\kappa) \geq k-1$. Further, $\kappa_{1}=\lambda_{2}+1$ or $\lambda_{1}+1$, depending on the relation between $\kappa_{1}$ and $\ell(\kappa)$. In either case, by the characterization of cycle partitions, we can conclude $\kappa_{1} \geq k$. Therefore, by Corollary 1 , no $\kappa \vdash n$ with $D_{P}(\kappa)=\lambda$ can be a GE-partition. 
We deal with some small values of $n$ before proceeding to general arguments. Note that there are no GE-partitions for $n=1,2$. For $n=3$, the GE-partition $\left(1^{3}\right)$ has preperiod length 2 to the unique cycle partition $(2,1)$. For $n=4$, the GE-partition $\left(1^{4}\right)$ has preperiod length 2 to the cycle partition $(3,1)$ (note $\Delta((3,1))=(1,0,0)$ ). For $n=5$, the GE-partition $\left(2,1^{3}\right)$ has preperiod length 2 to the cycle partition $(3,2)$ (note $\Delta((3,2))=(1,1,0))$.

Case 1. Let $n=T_{k}$ for $k \geq 3$. Consider the three successive images under $D_{P}$ of $\left(k, \searrow, 4,2,1^{4}\right)$ with length $k+2$, which is a GE-partition:

$$
\begin{aligned}
D_{P}^{3}((k, & \left.\left.\searrow, 4,2,1^{4}\right)\right) \\
& =D_{P}^{2}((k+2, k-1, \searrow, 3,1)) \text { of length } k-1 \\
& =D_{P}((k+1, k-1, \searrow, 2)) \text { of length } k-1 \\
& =(k, \searrow, 1) \text { of length } k,
\end{aligned}
$$

the unique cycle partition. To show that 3 is the minimal preperiod length between a GE-partition and the cycle partition, we need to show that there are no GE-partitions 2 applications of $D_{P}$ away from the cycle. By Lemma 1, the only partition whose image is $(k, \searrow, 1)$, other than itself, is $(k+1, k-1, \searrow, 2)$ with length $k-1$; for an example when $n=6$, see Fig. 6. Again, by Lemma 1, this partition has three preimages under $D_{P}$, namely

$$
\begin{aligned}
& (k, \searrow, 3,1,1,1) \text { of length } k+1, \\
& (k+2, k-1, \searrow, 3,1) \text { of length } k-1, \text { and } \\
& (k+2, k, k-2, \searrow, 3) \text { of length } k-2 .
\end{aligned}
$$

None of these are GE-partitions.

For the subsequent cases where $n=T_{k}+r$ with $1 \leq r \leq k$, every cycle partition $\lambda$ of $n$ has $\Delta(\lambda)=\left(\delta_{k}, \ldots, \delta_{0}\right)$ with at least one 0 and at least one 1 . Since partitions in the cycle are related by cycling this binary vector, we choose to work with a cycle partition whose $\Delta$ satisfies $\delta_{k}=1$ and $\delta_{0}=0$. We consider four cases determined by $\delta_{k-1}$ and $\delta_{k-2}$.

Cases 2-5 establish the initial statement of the theorem, that given a cycle of partitions for any $n \geq 3$, there is a GE-partition at most 3 applications of $D_{P}$ away from a partition in the cycle. Minimality for these cases is discussed before case 6. For cases 2-5, we expand our notation to represent $\left(k+\delta_{k}, \ldots, 1+\delta_{1}, \delta_{0}\right)$ by $\left(k+\delta_{k}, \searrow, 1+\delta_{1}, \delta_{0}\right)$.

Case 2. $\Delta(\lambda)=\left(1,0,0, \delta_{k-3}, \ldots, \delta_{1}, 0\right)$. Consider the two successive images under $D_{P}$ of $\left(k, k-1+\delta_{k-3}, \searrow, 3+\delta_{1}, 1^{4}\right)$ with length $k+2$, which is a GE-partition:

$$
\begin{aligned}
D_{P}^{2}((k, & \left.\left.k-1+\delta_{k-3}, \searrow, 3+\delta_{1}, 1^{4}\right)\right) \\
& =D_{P}\left(\left(k+2, k-1, k-2+\delta_{k-3}, \searrow, 2+\delta_{1}\right)\right) \text { of length } k-1 \\
& =\left(k+1, k-1, k-2, k-3+\delta_{k-3}, \searrow, 1+\delta_{1}\right) \text { of length } k .
\end{aligned}
$$

This results in the cycle partition $\lambda$ with $\Delta(\lambda)$ as specified. Since we showed earlier that there are no GE-partitions whose image under $D_{P}$ is a cycle partition, this shows the minimal preperiod length in this case is 2 . 
Case 3. $\Delta(\lambda)=\left(1,0,1, \delta_{k-3}, \ldots, \delta_{1}, 0\right)$. Consider the three successive images under $D_{P}$ of $\left(k+\delta_{k-3}, \searrow, 4+\delta_{1}, 2,2,1^{4}\right)$ with length $k+3$, which is a GE-partition:

$$
\begin{aligned}
D_{P}^{3}((k & \left.\left.+\delta_{k-3}, \searrow, 4+\delta_{1}, 2,2,1^{4}\right)\right) \\
& =D_{P}^{2}\left(\left(k+3, k-1+\delta_{k-3}, \searrow, 3+\delta_{1}, 1,1\right)\right) \text { of length } k \\
& =D_{P}\left(\left(k+2, k, k-2+\delta_{k-3}, \searrow, 2+\delta_{1}\right)\right) \text { of length } k-1 \\
& =\left(k+1, k-1, k-1, k-3+\delta_{k-3}, \searrow, 1+\delta_{1}\right) \text { of length } k .
\end{aligned}
$$

This results in the cycle partition $\lambda$ with $\Delta(\lambda)$ as specified. (For $n=8$, this corresponds to the path from $\left(2,2,1^{4}\right)$ to $(4,2,2)$ in the smaller component shown in Fig. 4.)

Case 4. $\Delta(\lambda)=\left(1,1,0, \delta_{k-3}, \ldots, \delta_{1}, 0\right)$. Consider the two successive images under $D_{P}$ of $\left(k, k-1+\delta_{k-3}, \searrow, 3+\delta_{1}, 2,1^{3}\right)$ with length $k+2$, which is a GE-partition:

$$
\begin{aligned}
D_{P}^{2}((k, & \left.\left.k-1+\delta_{k-3}, \searrow, 3+\delta_{1}, 2,1^{3}\right)\right) \\
& =D_{P}\left(\left(k+2, k-1, k-2+\delta_{k-3}, \searrow, 2+\delta_{1}, 1\right)\right) \text { of length } k \\
& =\left(k+1, k, k-2, k-3+\delta_{k-3}, \searrow, 1+\delta_{1}\right) \text { of length } k .
\end{aligned}
$$

This results in the cycle partition $\lambda$ with $\Delta(\lambda)$ as specified. (For $n=8$, this corresponds to the path from $\left(3,2,1^{3}\right)$ to $(4,3,1)$ in the larger component shown in Fig. 4.)

Case 5. $\Delta(\lambda)=\left(1,1,1, \delta_{k-3}, \ldots, \delta_{1}, 0\right)$. Consider the three successive images under $D_{P}$ of $\left(k+\delta_{k-3}, \searrow, 4+\delta_{1}, 3,2,1^{4}\right)$ with length $k+3$, which is a GE-partition:

$$
\begin{aligned}
D_{P}^{3}((k & \left.\left.+\delta_{k-3}, \searrow, 4+\delta_{1}, 3,2,1^{4}\right)\right) \\
& =D_{P}^{2}\left(\left(k+3, k-1+\delta_{k-3}, \searrow, 3+\delta_{1}, 2,1\right)\right) \text { of length } k \\
& =D_{P}\left(\left(k+2, k, k-2+\delta_{k-3}, \searrow, 2+\delta_{1}, 1\right)\right) \text { of length } k \\
& =\left(k+1, k, k-1, k-3+\delta_{k-3}, \searrow, 1+\delta_{1}\right) \text { of length } k .
\end{aligned}
$$

This results in the cycle partition $\lambda$ with $\Delta(\lambda)$ as specified.

These cases show that every cycle of partitions can be reached with at most 3 applications of the $D_{P}$ map from some GE-partition, so that no cycle is isolated. It remains to show that, for $n=T_{k}+r$ with $1 \leq r \leq k$, the minimal preperiod length from a GE-partition to some cycle partition is 2 . The case $r=1$ is covered by case 2 above, and $r=2$ by case 4 . While some other cases, depending on $k$ and $r$, are covered by those two arguments, not every $n$ has a cycle partition $\lambda$ with $\Delta(\lambda)$ covered by cases 2 and 4 . While there are partition cycles for which the bounds given in cases 3 and 5 are sharp (such as the examples mentioned in $P(8)$ ), the next case shows that every $n$ has some cycle partition with preperiod length 2 .

Case 6. We now have $n=T_{k}+r$ with $3 \leq r \leq k$. Consider the two successive images under $D_{P}$ of $\left(k, \searrow, r, r, \searrow, 3,1^{3}\right)$ with length $k+2$, which is a GE-partition:

$$
\begin{aligned}
D_{P}^{2}((k, & \left.\left.\searrow, r, r, \searrow, 3,1^{3}\right)\right) \\
& =D_{P}((k+2, k-1, \searrow, r-1, r-1, \searrow, 2)) \text { length } k \\
& =(k+1, k, k-2, \searrow, r-2, r-2, \searrow, 1) \text { length } k+1 .
\end{aligned}
$$


This results in the cycle partition $\lambda$ with $\Delta(\lambda)=\left(1,1,0^{k-r+1}, 1^{r-2}\right)$.

The proof of Theorem 1 contributes to the program of understanding all GE-partition preperiod lengths. Earlier work has focused primarily on maximal preperiod lengths. For $n=T_{k}$, the maximal preperiod length is $k(k-1)$ [10], attained by the GE-partition $(k-1, k-1, \searrow, 1,1)[6]$. Various bounds on maximal preperiod lengths for other $n$ are given in [7] and [9]. Minimal preperiod lengths for the case $n=T_{k}$ are determined in [6], which also considers various intermediate preperiod lengths when $n=T_{k}$.

Having established that $G E_{P}(n)$ is a sufficient starting set to determine the entire structure of $P(n)$, we want to know its size relative to $P(n)$. We have not found an exact formula, but we show the number of GE-partitions is bounded below by an established sequence that can be described in terms of $p(n)=|P(n)|$.

Another notation for partitions simplifies the following discussion. The Frobenius symbol of a partition $\lambda$ is a $2 \times k$ array of nonnegative integers

$$
\left(\begin{array}{lll}
a_{1} & \ldots & a_{k} \\
b_{1} & \ldots & b_{k}
\end{array}\right)
$$

where $k$ is the number of dots on the diagonal of the Ferrers diagram of $\lambda, a_{i}$ is the number of dots to the right of the $i$ th diagonal dot, and $b_{i}$ is the number of dots under the $i$ th diagonal dot. Fig. 5 includes some examples. Notice that the numbers in each row on the Frobenius symbol must be strictly decreasing. This notation highlights conjugation, as the Frobenius symbols of $\lambda$ and $\lambda^{\prime}$ simply have the two rows interchanged.

It is also easy to read from a partition's Frobenius symbol whether it is a GE-partition: $\lambda_{1}=a_{1}+1$ and $\ell(\lambda)=b_{1}+1$, so the characterization of Lemma 1 becomes $\lambda$ is a GEpartition exactly when $a_{1}-b_{1} \leq-2$.

Theorem 2. The number of GE-partitions in $P(n)$ is at least the number of conjugate pairs in $P(n-1)$.

Proof. We construct a one-to-one map from conjugate pairs of $P(n-1)$ into $G E_{P}(n)$.

Let $\lambda \vdash n-1$ satisfy $\lambda \neq \lambda^{\prime}$. Without loss of generality, assume that the entries of the Frobenius symbol for $\lambda$ satisfy $a_{1}=b_{1}, \ldots, a_{j}=b_{j}, a_{j+1}<b_{j+1}$, i.e., $\lambda$ is the "more vertical' partition of the conjugate pair. We construct $\mu \vdash n$ from $\lambda$ as follows, using the entries of the Frobenius symbol of $\lambda$ and the parameter $j$ (which may be 0). Let

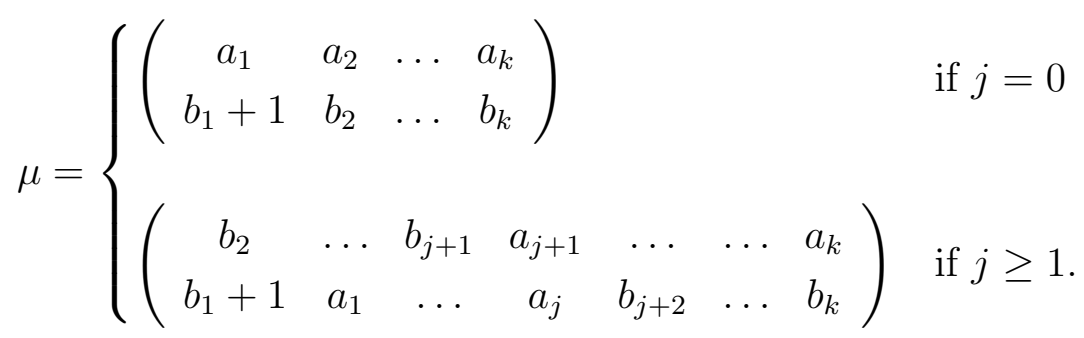

In words, $\mu$ is constructed by adding a dot to the first column of $\lambda$ and swapping the first $j$ horizontal arms from the diagonal with the second to $(j+1)$ st vertical arms. See Fig. 5 for an example. 


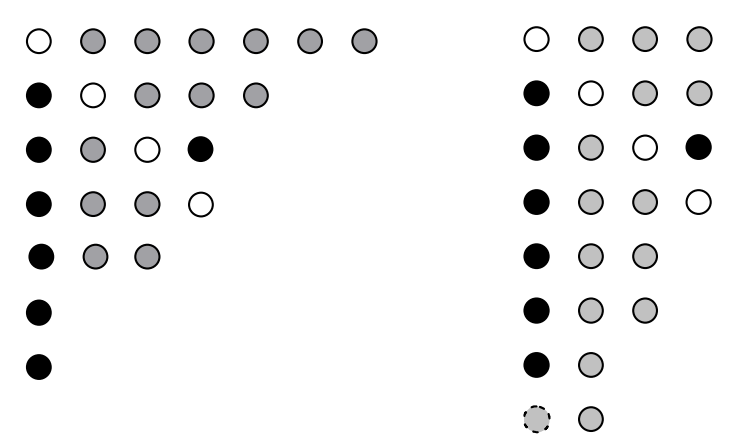

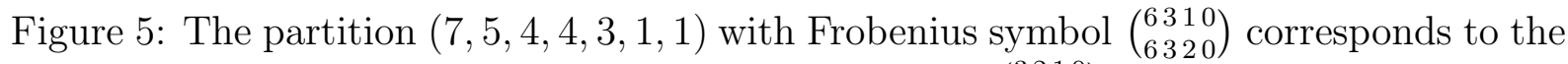
GE-partition $(4,4,4,4,3,3,2,2)$ with Frobenius symbol $\left(\begin{array}{llll}3 & 2 & 1 & 0 \\ 7 & 6 & 3 & 0\end{array}\right)$.

First, we show that the array is the Frobenius symbol of a partition. The $j=0$ case is clear. For the $j \geq 1$ cases, the entries of the first row are strictly decreasing since $\lambda$ was chosen to have $b_{j+1}>a_{j+1}$. For the second row, $b_{1}+1=a_{1}+1>a_{1}$ and $a_{j}=b_{j}>b_{j+2}$. Since one dot has been added, we have $\mu \vdash n$.

Next, we show that $\mu \in G E_{P}(n)$. For the $j=0$ case, by assumption, $b_{1}>a_{1}$, i.e., $a_{1}-b_{1} \leq-1$. The difference of the numbers in the first column of the Frobenius symbol for $\mu$ is then $a_{1}-\left(b_{1}+1\right)=a_{1}-b_{1}-1 \leq-2$. For the $j \geq 1$ cases, we know $b_{1} \geq b_{2}+1$, from which $b_{2}-\left(b_{1}-1\right) \leq-2$ as well. Therefore $\mu$ is a GE-partition.

Notice that the GE-partitions constructed in this way, from $\lambda$ with $j \geq 2$, have $b_{3}=a_{1}$ in their Frobenius symbol, since these correspond to $a_{2}$ and $b_{2}$ of $\lambda$. This is not true of GE-partitions in general and shows the limitations of the map: we show next that it is an injection, but it is not a bijection. The smallest GE-partition not in its image is

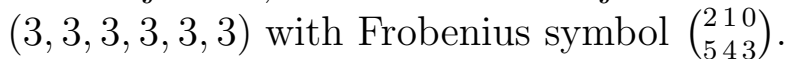

For the inverse map, let $\mu \in G E_{P}(n)$. If $b_{1}>b_{2}+1$, let $j=0$. If $b_{1}=b_{2}+1$ and $b_{3} \neq a_{1}$, let $j=1$. Otherwise, let $j$ be the greatest index for which $b_{3}=a_{1}, \ldots$, $b_{j+1}=a_{j-1}$. We construct $\lambda \vdash n-1$ as follows.

$$
\lambda=\left\{\begin{array}{ccccc}
\left(\begin{array}{ccccc}
a_{1} & a_{2} & \ldots & a_{k} \\
b_{1}-1 & b_{2} & \ldots & b_{k}
\end{array}\right) & & & \text { if } j=0 \\
\left(\begin{array}{ccccccc}
b_{2} & \ldots & b_{j+1} & a_{j+1} & \ldots & \ldots & a_{k} \\
b_{1}-1 & a_{1} & \ldots & a_{j} & b_{j+2} & \ldots & b_{k}
\end{array}\right) & \text { if } j \geq 1
\end{array}\right.
$$

In words, $\lambda$ is constructed by removing a dot from the first column of $\mu$ and the same swapping as in the previous map. Since $\mu$ is a GE-partition, $a_{1}-b_{1} \leq-2$, so $b_{1}-1>a_{1}$. Notice that if $j \geq 2$ and $b_{j+2}>a_{j}$, then the proposed array is not a Frobenius symbol, so the map is not defined for such $\mu$. Otherwise, a verification similar to the preceding shows that this is the Frobenius symbol for $\lambda \in P(n-1)$ with $\lambda \neq \lambda^{\prime}$ and it is clear that the two maps described are inverses. 
As mentioned, the map first fails to be a bijection at $n=18$, as there are 146 conjugate pairs in $P(17)$ and 147 GE-partitions of 18. For $n=60$, the injection misses 6,143 of the 421,957 GE-partitions, less than 1.5\%.

The number of pairs of conjugate partitions $\lambda \neq \lambda^{\prime}$, first documented in [13], is 1,1 , $2,3,5,7,10,14,20,27,37,49,66,86,113,146, \ldots$, starting from $n=2$. Therefore there at least that many GE-partitions of $n$, starting from $n=3$. With an observation by Jovovic in [14], we have

$$
\left|G E_{P}(n)\right| \geq p(n-3)-p(n-9)+p(n-19)-p(n-33)+\cdots+(-1)^{k+1} p\left(n-1-2 k^{2}\right)
$$

for the largest $k$ such that $n-1-2 k^{2} \geq 0$. This suggests that $|G E(n)|$ is on the order of $p(n-3)$, a significant improvement over $p(n)$ for large $n$.

\section{Compositions}

Although there are generally many more compositions than partitions for a fixed $n$, compositions are more structured in many ways. For instance, while the Hardy-RamanujanRademacher formula for $p(n)$ has "an infinite series involving $\pi$, square roots, complex roots of unity, and derivatives of hyperbolic functions," [2], the analogous $c(n)=|C(n)|$ is simply $2^{n-1}$. We give one of MacMahon's proofs of this formula [11], since ideas in the proof will be used later.

Proposition 1. $c(n)=2^{n-1}$.

Proof. Between each digit of $1^{n}$, place a + or $\oplus$. We show that the set of all possible resulting sequences is in bijection to $C(n)$. Two digits with a $\oplus$ between them are summed, while digits separated by + remain different parts. For example,

$$
1+1 \oplus 1 \oplus 1+1 \oplus 1 \longrightarrow 1+3+2
$$

The inverse is clear. Since there are $n-1$ binary decisions to create the sequence, the claim follows.

Similarly, the dynamics on $C(n)$ determined by $D_{C}$, while having a directed graph with more vertices than its partition analog, is often easier to analyze. For instance, since the map $D_{C}$ requires no reordering, every preimage of a composition $\lambda$ has the same length, namely $\lambda_{1}$.

The structure of the directed graph representing the map $D_{C}$ on compositions of $n$ is related to the corresponding directed graph for $D_{P}$ on partitions of $n$. For comparison, the state diagrams of $D_{P}$ and $D_{C}$ for $n=6$ appear in Fig. 6 . The boxed entries in Fig. 6 represent equivalence classes of compositions that have the same partition representation. Compositions in an equivalence class may or may not have the same image under $D_{C}$; this depends on the distribution of ones in the compositions and the order of the parts greater than 1. 


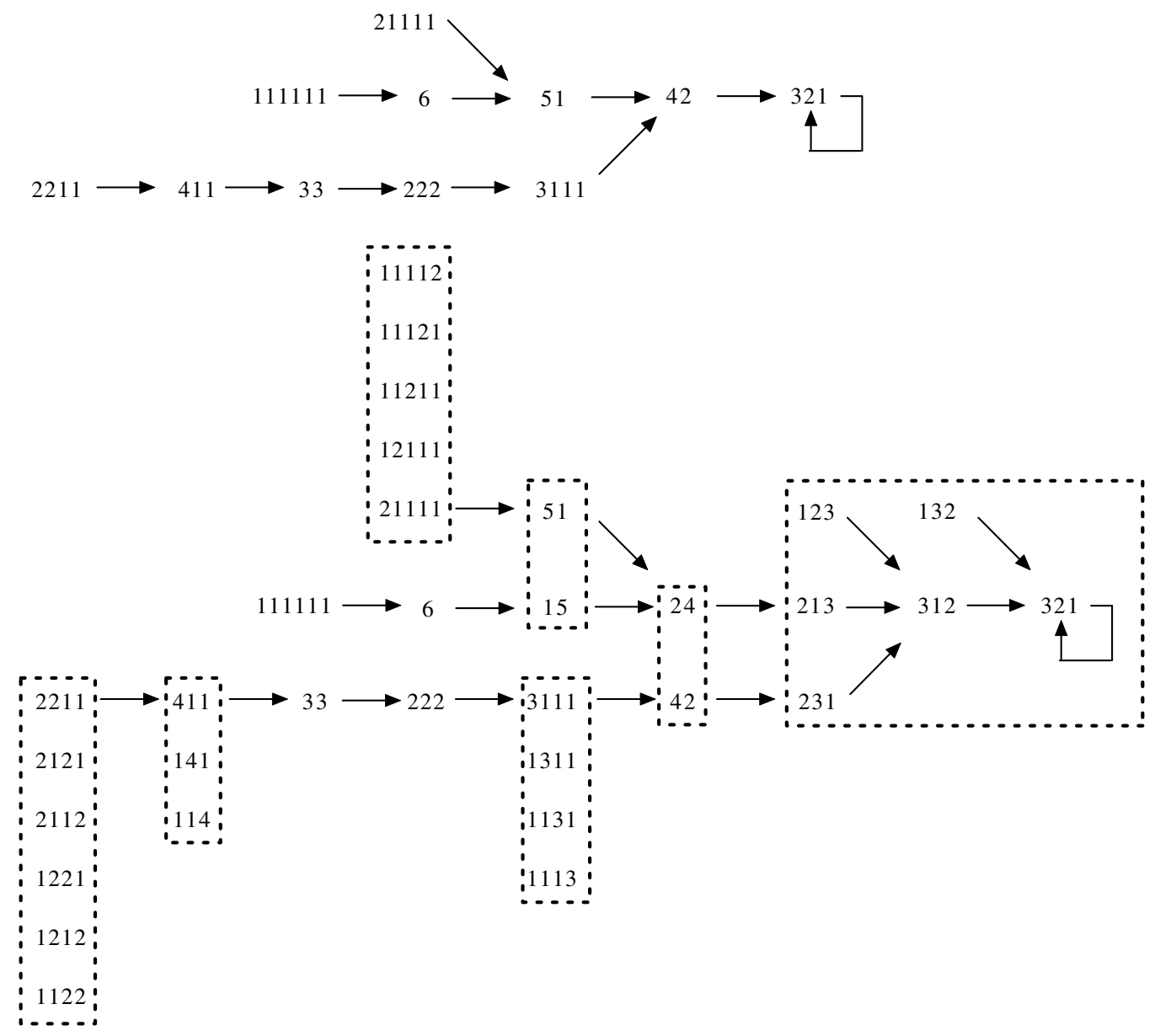

Figure 6: Expanding the directed graph for the shift map on partitions of 6 to the map on compositions of 6 .

Results for compositions are often similar to those for partitions, and sometimes simpler. First, we show that GE-compositions have the same characterization as GEpartitions, namely that the length of the composition is greater than the size of the first part plus 1. In this setting, this result is a corollary to the following proposition. First, we make another definition.

Definition 4. We say that compositions $\theta$ and $\kappa$ are related by a partial permutation if they correspond to the same partition and the integer parts greater than 1 appear in the same relative order.

Lemma 2. Compositions $\theta$ and $\kappa$ are related by a partial permutation if and only if $D_{C}(\theta)=D_{C}(\kappa)$.

Proof. If $\theta$ and $\kappa$ are related by a partial permutation, then the application of $D_{C}$ removes any and all ones from $\theta$ and $\kappa$ and puts an initial row of length $\ell(\theta)=\ell(\kappa)$ on the image 
compositions. The remaining rows of the images are one less than the parts of $\theta$ and $\kappa$ greater than 1 . Since those parts are in the same order in $\theta$ and $\kappa$, the images are identical.

Assume that $D_{C}(\theta)=D_{C}(\kappa)=\lambda$. By the definition of $D_{C}$, it follows that $\ell(\theta)=$ $\ell(\kappa)=\lambda_{1}$. Also, $\theta$ and $\kappa$ each have $\lambda_{1}-(\ell(\lambda)-1)$ ones. From the parts $\lambda_{2}, \ldots, \lambda_{k}$ in $\lambda$, we know that $\lambda_{2}+1, \ldots, \lambda_{k}+1$ must appear in the same relative order in $\theta$ and $\kappa$. Hence $\theta$ and $\kappa$ are related by a partial permutation (the ones may appear in any of the positions of $\theta$ and $\kappa$ without effecting their images under $D_{C}$ ).

Proposition 2. A composition $\lambda$ has indegree $\left(\begin{array}{c}\lambda_{1} \\ \ell(\lambda)-1\end{array}\right)$.

Proof. Suppose $\kappa$ is a preimage of $\lambda$, i.e., $D_{C}(\kappa)=\lambda$. Then $\lambda_{1}=\ell(\kappa)$ by the definition of $D_{C}$. Also, $\kappa$ contains $\ell(\kappa)-(\ell(\lambda)-1)$ ones. By Lemma 2, any partial permutation of $\kappa$ maps to $\lambda$ under $D_{C}$. There are $\left(\begin{array}{c}\lambda_{1} \\ \ell(\lambda)-1\end{array}\right)$ partial permutations of $\kappa$, because that counts the ways to select the positions of the parts greater than 1 .

Corollary 2. A composition $\lambda$ is a GE-composition if and only if $\ell(\lambda)>\lambda_{1}+1$.

The shift map on compositions has been studied in [9] where it is called "Carolina solitaire." We cite their primary results before going on to new material. Griggs and Ho [9] show that $\lambda \models n$ is cyclic under $D_{C}$ if and only if the parts are in decreasing order and $\lambda$ is cyclic under $D_{P}$. Hence, the cycle structure and corresponding number of components of the directed graph representation of $D_{C}$ are the same as for the shift map on partitions. Griggs and Ho provide a lower bound for the maximal preperiod length from a GE-composition to a cycle composition and conjecture that this bound is tight. Analogous to Theorem 1, we prove that there are no stand alone cycles for the shift map on compositions.

Lemma 3. If $D_{C}(\kappa)=\lambda$ with $\lambda_{1}>2$ and $\kappa_{i}=1$ for some $i$, then there exists at least one GE-composition $\theta$ such that $D_{C}(\theta)=\lambda$.

Proof. By the previous lemma, we know all partial permutations of $\kappa$ are preimages of $\lambda$. At least one of these has initial part 1 ; call this composition $\theta$. Since

$$
\ell(\theta)=\lambda_{1}>2=\theta_{1}+1,
$$

we know by Corollary 2 that $\theta$ is a GE-composition.

Theorem 3. For $n \geq 3$, every cycle composition $\lambda \in C(n)$ satisfies $D_{C}^{m}(\kappa)=\lambda$ for some $\kappa \in G E_{C}(n)$ and $m \geq 1$.

Proof. We prove that, for $n \geq 4$, the minimum preperiod length for a GE-composition of $n$ is 1 . For smaller $n$, there are no GE-compositions for $n=1,2$, and $\left(1^{3}\right)$ is the only GE-composition for $n=3$, which is distance 2 from the cycle composition $(2,1)$.

From the characterization of cycle compositions, we know that, for any $n \geq 4$, every cycle contains at least one cycle composition $\kappa$ with final part 1 and $\ell(\kappa)>2$. Then $\lambda$ determined by $D_{C}(\kappa)=\lambda$, another cycle composition, has $\lambda_{1}>2$. By the previous lemma, there is at least one GE-composition $\theta$, a partial permutation of $\kappa$, whose image is $\lambda$. 
For example, in Fig. 6, the GE-composition $(1,3,2)$ has preperiod 1. So while maximal preperiod lengths for compositions are longer than for partitions, minimal preperiod lengths are shorter.

Again, having established that $G E_{C}(n)$ is a sufficient starting set to determine the entire structure of $C(n)$, we want to count the GE-compositions. Unlike the situation with partitions, we establish an exact formula. In fact, we prove two formulas for the number of GE-compositions, $\left|G E_{C}(n)\right|$.

Theorem 4. $\left|G E_{C}(n)\right|=2^{n-1}-F_{n+1}$, where $F_{i}$ are the Fibonacci numbers determined by $F_{1}=F_{2}=1$ and $F_{n}=F_{n-1}+F_{n-2}$ for $n \geq 3$.

Proof. We count the number of compositions of $n$ that are in the image of $D_{C}$ using induction. Let $I_{n}$ denote the set of compositions that have at least one preimage under $D_{C}$ (equivalently, those with positive indegree in the state diagram). By inspection, $I_{1}=\{(1)\}$ and $I_{2}=\{(2),(1,1)\}$, so that $\left|I_{1}\right|=F_{2}$ and $\left|I_{2}\right|=F_{3}$.

Assume that $\left|I_{n-1}\right|=F_{n}$ and $\left|I_{n-2}\right|=F_{n-1}$. We define maps from $I_{n-1}$ and $I_{n-2}$ into $I_{n}$ that will lead to the conclusion that $\left|I_{n}\right|=\left|I_{n-1}\right|+\left|I_{n-2}\right|$.

For each $\lambda=\left(\lambda_{1}, \ldots, \lambda_{k}\right) \in I_{n-1}$, define

$$
a(\lambda)=\left(\lambda_{1}, \ldots, \lambda_{k-1}, \lambda_{k}+1\right) .
$$

Notice that $a(\lambda) \models n$ and $\ell(a(\lambda))=k$. Since $\lambda \in I_{n-1}$, we know by Corollary 2 that $\lambda_{1} \geq k-1$, which shows that $a(\lambda) \in I_{n}$. The map $a$ is clearly bijective.

For each $\mu=\left(\mu_{1}, \ldots, \mu_{j}\right) \in I_{n-2}$, define

$$
b(\mu)=\left(\mu_{1}+1, \mu_{2}, \ldots, \mu_{j}, 1\right) .
$$

Notice that $b(\mu) \models n$ and $\ell(b(\mu))=j+1$. Since $\mu \in I_{n-2}$, we know by Corollary 2 that $\mu_{1} \geq j-1$. Therefore, $\mu_{1}+1 \geq j$, which shows that $b(\mu) \in I_{n}$. Again, $b$ is injective. See Fig. 7 for examples of applications of these maps.
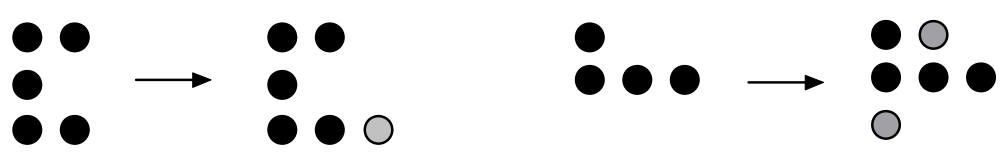

Figure $7: a((2,1,2))=(2,1,3)$ and $b((1,3))=(2,3,1)$.

Writing $a\left(I_{n-1}\right)$ and $b\left(I_{n-2}\right)$ for these sets of compositions of $n$, we have $a\left(I_{n-1}\right) \cup$ $b\left(I_{n-2}\right) \subseteq I_{n}$.

We show that $I_{n} \subseteq a\left(I_{n-1}\right) \cup b\left(I_{n-2}\right)$. Consider an arbitrary $\nu=\left(\nu_{1}, \ldots, \nu_{h}\right) \in I_{n}$. By Corollary 2, we know $\nu_{1} \geq h-1$. If $\nu_{h} \geq 2$, then $\left(\nu_{1}, \ldots, \nu_{h-1}, \nu_{h}-1\right) \in I_{n-1}$ since $\nu_{1} \geq h-1$, so that $\nu \in a\left(I_{n-1}\right)$. If $\nu_{h}=1$, then $\left(\nu_{1}-1, \nu_{2}, \ldots, \nu_{h-1}\right) \in I_{n-2}$ since $\nu_{1}-1 \geq h-2$, so that $\nu \in b\left(I_{n-2}\right)$. Therefore $I_{n}=a\left(I_{n-1}\right) \cup b\left(I_{n-2}\right)$.

By looking at the last entry of the compositions, clearly $a\left(I_{n-1}\right) \cap b\left(I_{n-2}\right)=\emptyset$. Therefore

$$
\left|I_{n}\right|=\left|a\left(I_{n-1}\right)\right|+\left|b\left(I_{n-2}\right)\right|=\left|I_{n-1}\right|+\left|I_{n-2}\right|=F_{n}+F_{n-1}=F_{n+1} .
$$

Since there are $2^{n-1}$ total compositions of $n,\left|G E_{C}(n)\right|=2^{n-1}-F_{n+1}$. 
The second formula for $\left|G E_{C}(n)\right|$ comes from a result on the number of compositions of a given length. Before we proceed, we need a few smaller results. The first lemma is another result of MacMahon [11].

Lemma 4. There are $\left(\begin{array}{c}n-1 \\ k-1\end{array}\right)$ compositions of $n$ of length $k$ where $k \leq n$.

Proof. Using the bijection of Proposition 1, these compositions correspond to sequences of $n$ ones where exactly $k-1$ of the $n-1$ separators are + and the rest are $\oplus$.

Lemma 5. There are $\left(\begin{array}{c}n-j-1 \\ k-2\end{array}\right)$ compositions of $n$ of length $k$ with first part $j<n$

Proof. Because $j<n$, it follows that $k \geq 2$. Using the bijection from Proposition 1 , these compositions correspond to sequences of $n$ ones where the first $j-1$ separators are $\oplus$ and the next separator is + . To have $k-1$ more parts, exactly $k-2$ of the remaining $n-j-1$ separators need to be + .

In the following theorem and discussion, we will let $\lfloor\cdot\rfloor$ represent the floor function and $\lceil\cdot 7$ represent the ceiling function.

\section{Theorem 5 .}

$$
\left|G E_{C}(n)\right|=\sum_{j=1}^{\left\lfloor\frac{n}{2}\right\rfloor}\left(2^{n-j-1}-\sum_{k=2}^{j+1}\left(\begin{array}{c}
n-j-1 \\
k-2
\end{array}\right)\right) .
$$

Proof. First, we show that the first part of a GE-composition cannot be greater than $\left\lfloor\frac{n}{2}\right\rfloor$. Let $\mu=\left(\mu_{1}, \ldots, \mu_{k}\right) \models n$ be a GE-composition. If $\mu_{1}>\left\lfloor\frac{n}{2}\right\rfloor$, then $k \leq n-\mu_{1}+1<$ $n-\left\lfloor\frac{n}{2}\right\rfloor+1$. It follows that

$$
\ell(\mu)=k \leq n-\left\lfloor\frac{n}{2}\right\rfloor \leq\left\lfloor\frac{n}{2}\right\rfloor+1<\mu_{1}+1,
$$

contradicting Corollary 2. Hence, $\mu_{1} \leq\left\lfloor\frac{n}{2}\right\rfloor$.

Let $\lambda=\left(j, \lambda_{2}, \ldots, \lambda_{k}\right) \models n$ with $j \leq\left\lfloor\frac{n}{2}\right\rfloor$ and denote $\lambda^{*}=\left(\lambda_{2}, \ldots, \lambda_{k}\right) \models n-j$. By Lemma 4 , there are $\left(\begin{array}{c}n-j-1 \\ k-2\end{array}\right)$ compositions $\lambda^{*}$ in $C(n-j)$ of length $k-1$. Because $c(n-j)=2^{n-j-1}$ by Proposition 1, there are $2^{n-j-1}-\left(\begin{array}{c}n-j-1 \\ k-2\end{array}\right)$ compositions of length $k$ in $C(n)$ with first part $j$.

By Corollary 2, if $k \leq \lambda_{1}+1$, then $\lambda$ is not a GE-composition. Summing over the values of $k \leq j+1$ yields the number of compositions of $n$ with $\lambda_{1}=j$ that are not GE-compositions, namely $\sum_{k=2}^{j+1}\left(\begin{array}{c}n-j-1 \\ k-2\end{array}\right)$. Notice that $k \geq 2$ because $\lambda_{1}=j \leq\left\lfloor\frac{n}{2}\right\rfloor$, so $\lambda$ has at least 2 parts. Thus there are $2^{n-j-1}-\sum_{k=2}^{j+1}\left(\begin{array}{c}n-j-1 \\ k-2\end{array}\right)$ GE-compositions of $n$ with $\lambda_{1}=j$. Because $j$ can range from 1 to $\left\lfloor\frac{n}{2}\right\rfloor$, the number of GE-compositions is

$$
\sum_{j=1}^{\left\lfloor\frac{n}{2}\right\rfloor}\left(2^{n-j-1}-\sum_{k=2}^{j+1}\left(\begin{array}{c}
n-j-1 \\
k-2
\end{array}\right)\right) .
$$


Together, these two results give a round-about combinatorial proof of the following Fibonacci identity:

$$
\begin{aligned}
F_{n+1} & =2^{n-1}-\sum_{j=1}^{\left\lfloor\frac{n}{2}\right\rfloor}\left(2^{n-j-1}-\sum_{k=2}^{j+1}\left(\begin{array}{c}
n-j-1 \\
k-2
\end{array}\right)\right) \\
& =2^{\left\lceil\frac{n}{2}\right\rceil-1}+\sum_{j=1}^{\left\lfloor\frac{n}{2}\right\rfloor} \sum_{i=0}^{j-1}\left(\begin{array}{c}
n-j-1 \\
i
\end{array}\right) .
\end{aligned}
$$

This can also be derived using two well-known Fibonacci identities,

$$
F_{n+1}-1=\sum_{i=1}^{n-1} F_{i} \quad \text { and } \quad F_{n+1}=\sum_{i=0}^{\left\lfloor\frac{n}{2}\right\rfloor}\left(\begin{array}{c}
n-i \\
i
\end{array}\right)
$$

(both proved combinatorially in [5]), the binomial theorem, and a simple application of geometric series:

$$
\begin{aligned}
F_{n+1} & =1+\sum_{i=1}^{n-1} F_{i} \\
& =1+\sum_{i=1}^{n-1} \sum_{h=0}^{\left\lfloor\frac{i-1}{2}\right\rfloor}\left(\begin{array}{c}
i-h-1 \\
h
\end{array}\right) \\
& =1+\sum_{h=0}^{\left\lceil\frac{n}{2}\right\rceil-2} \sum_{i=0}^{h}\left(\begin{array}{c}
h \\
i
\end{array}\right)+\sum_{h=\left\lceil\frac{n}{2}\right\rceil-1}^{n-2} \sum_{i=0}^{n-h-2}\left(\begin{array}{c}
h \\
i
\end{array}\right) \\
& =1+\sum_{h=0}^{\left\lceil\frac{n}{2}\right\rceil-2} 2^{h}+\sum_{j=1}^{\left\lfloor\frac{n}{2}\right\rfloor} \sum_{i=0}^{j-1}\left(\begin{array}{c}
n-j-1 \\
i
\end{array}\right) \\
& =1+2^{\left\lceil\frac{n}{2}\right\rceil-1}-1+\sum_{j=1}^{\left\lfloor\frac{n}{2}\right\rfloor} \sum_{i=0}^{j-1}\left(\begin{array}{c}
n-j-1 \\
i
\end{array}\right) .
\end{aligned}
$$

In addition to exact formulas for $\left|G E_{C}(n)\right|$, we can further our investigation of GEcompositions by counting how many have a given indegree. We do not arrive at a formula, rather an algorithm. From Proposition 2, counting the number of compositions with a given indegree requires determining all of the ways that the indegree can be written as a binomial coefficient with certain constraints. This generalizes Lemma 5.

Proposition 3. All of the $\left(\begin{array}{c}n-j-1 \\ k-2\end{array}\right)$ compositions of $n$ of length $k$ with first part $j<n$ have indegree $\left(\begin{array}{c}j \\ k-1\end{array}\right)$.

Proof. By Proposition 2, the indegree of a composition depends only on its length and first part, so these compositions all have indegree $\left(\begin{array}{c}j \\ k-1\end{array}\right)$. 


$$
\begin{aligned}
& \left(\begin{array}{l}
1 \\
1
\end{array}\right): 1 \\
& \left(\begin{array}{l}
2 \\
1
\end{array}\right): 1 \quad\left(\begin{array}{l}
2 \\
2
\end{array}\right): 5 \\
& \left(\begin{array}{l}
3 \\
1
\end{array}\right): 1 \quad\left(\begin{array}{l}
3 \\
2
\end{array}\right): 4 \quad\left(\begin{array}{l}
3 \\
3
\end{array}\right): 6 \\
& \left(\begin{array}{l}
4 \\
1
\end{array}\right): 1 \quad\left(\begin{array}{l}
4 \\
2
\end{array}\right): 3 \quad\left(\begin{array}{l}
4 \\
3
\end{array}\right): 3 \quad\left(\begin{array}{l}
4 \\
4
\end{array}\right): 1 \\
& \left(\begin{array}{l}
5 \\
1
\end{array}\right): 1 \quad\left(\begin{array}{l}
5 \\
2
\end{array}\right): 2 \quad\left(\begin{array}{l}
5 \\
3
\end{array}\right): 1 \\
& \left(\begin{array}{l}
6 \\
1
\end{array}\right): 1 \quad\left(\begin{array}{l}
6 \\
2
\end{array}\right): 1 \\
& \left(\begin{array}{l}
7 \\
1
\end{array}\right): 1
\end{aligned}
$$

Figure 8: Truncated Pascal's triangle containing the number of compositions of $n=8$ with the specific indegree as entries of the form indegree:number.

The proposition fails to consider length 1 compositions. There is a unique length 1 composition, $(n)$, which has indegree 1 since $D_{C}\left(\left(1^{n}\right)\right)=(n)$ and no other composition has length $n$. We apply this proposition to the compositions of $C(8)$.

Example 1. The indegrees for compositions of $n=8$. Proposition 3 suggests creating a form of Pascal's triangle where the entries are indegrees from a particular binomial coefficient paired with the number of compositions with that indegree. For example, the $\left(\begin{array}{l}4 \\ 2\end{array}\right)$ entry indicates the compositions with indegree 6 that have first part 4 and length 3 ; it is followed by a 3 since there are $\left(\begin{array}{c}8-4-1 \\ 3-1\end{array}\right)=\left(\begin{array}{l}3 \\ 2\end{array}\right)=3$ such compositions. The triangle for $C(8)$ is given in Fig. 8. The figure omits the GE-compositions, indegree 0 , and the composition (8), indegree 1. All of $C(8)$ is accounted for in Table 1, which collects the triangle entries with the same indegree. (The fact that binomial coefficients from different rows can have the same value, such as $\left(\begin{array}{l}4 \\ 2\end{array}\right)$ and $\left(\begin{array}{l}6 \\ 1\end{array}\right)$, prevents this approach from leading to a direct formula.)

\section{Suggestions for Further Work}

There are many remaining questions about the state diagrams induced by $D_{P}$ and $D_{C}$. First, we know from [4] that the diagrams have multiple components for any $n$ two or more away from the nearest triangular number - what are the sizes of those components? For partitions, we know that $D_{P}$ splits the 22 partitions in $P(8)$ into components of size 15 and 7 . For 12 and 13 , the splits are $77=45+32$ and $101=67+34$, respectively. The problem appears no easier for compositions: $D_{C}$ splits the 128 compositions in $C(8)$ into components of size 93 and 35.

While we have developed a procedure to determine the distribution of compositions in $C(n)$ according to their indegrees, we do not see an analogous approach to $P(n)$, even though there are fewer possible indegrees. Data up to $n=15$ are given in Table 2 .

Turning to GE-partitions, Theorem 2 gives a lower bound of their number; an exact formula is desired. Also, we know only the bounds for preperiod length. Theorem 1 


\begin{tabular}{|l|l|}
\hline Indegree & Number of Compositions \\
\hline 0 & $94\left(=2^{7}-F_{9}=128-34\right)$ \\
\hline $1\left[\right.$ from $\left(\begin{array}{l}1 \\
1\end{array}\right),\left(\begin{array}{l}2 \\
2\end{array}\right),\left(\begin{array}{l}3 \\
3\end{array}\right),\left(\begin{array}{l}4 \\
4\end{array}\right)$ and $\left.1^{8}\right]$ & $14(=1+5+6+1+1)$ \\
\hline $2\left[\right.$ from $\left.\left(\begin{array}{l}2 \\
1\end{array}\right)\right]$ & 1 \\
\hline $3\left[\right.$ from $\left(\begin{array}{l}3 \\
1\end{array}\right)$ and $\left.\left(\begin{array}{l}3 \\
2\end{array}\right)\right]$ & $5(=1+4)$ \\
\hline $4\left[\right.$ from $\left(\begin{array}{l}4 \\
1\end{array}\right)$ and $\left.\left(\begin{array}{l}4 \\
3\end{array}\right)\right]$ & $4(=1+3)$ \\
\hline $5\left[\right.$ from $\left.\left(\begin{array}{l}5 \\
1\end{array}\right)\right]$ & 1 \\
\hline $6\left[\right.$ from $\left(\begin{array}{l}6 \\
1\end{array}\right)$ and $\left.\left(\begin{array}{l}4 \\
2\end{array}\right)\right]$ & $4(=1+3)$ \\
\hline $7\left[\right.$ from $\left.\left(\begin{array}{l}7 \\
1\end{array}\right)\right]$ & 1 \\
\hline $10\left[\right.$ from $\left(\begin{array}{l}5 \\
2\end{array}\right)$ and $\left.\left(\begin{array}{l}5 \\
3\end{array}\right)\right]$ & $3(=2+1)$ \\
\hline $15\left[\right.$ from $\left.\left(\begin{array}{l}6 \\
2\end{array}\right)\right]$ & 1 \\
\hline
\end{tabular}

Table 1: The number of compositions of $n=8$ with specific indegrees.

\begin{tabular}{|c|c|c|c|c|c|c|c|c|c|c|c|c|c|c|c|}
\hline$i \backslash n$ & $\mathbf{1}$ & $\mathbf{2}$ & $\mathbf{3}$ & $\mathbf{4}$ & $\mathbf{5}$ & $\mathbf{6}$ & $\mathbf{7}$ & $\mathbf{8}$ & $\mathbf{9}$ & $\mathbf{1 0}$ & $\mathbf{1 1}$ & $\mathbf{1 2}$ & $\mathbf{1 3}$ & $\mathbf{1 4}$ & $\mathbf{1 5}$ \\
\hline $\mathbf{0}$ & 0 & 0 & 1 & 1 & 2 & 3 & 5 & $7: 5,2$ & 10 & 14 & 20 & $27: 17,10$ & $37: 24,13$ & 49 & 66 \\
\hline $\mathbf{1}$ & 1 & 2 & 1 & 3 & 3 & 5 & 5 & $8: 5,3$ & 11 & 15 & 18 & $26: 13,13$ & $32: 22,10$ & 44 & 55 \\
\hline $\mathbf{2}$ & & & 1 & 1 & 2 & 3 & 5 & $7: 5,2$ & 8 & 12 & 16 & $21: 13,8$ & $27: 18,9$ & 35 & 44 \\
\hline $\mathbf{3}$ & & & & & & & & & 1 & 1 & 2 & $3: 2,1$ & $5: 3,2$ & 7 & 11 \\
\hline
\end{tabular}

Table 2: The number of partitions of $n$ with specific indegree $i$, broken down by component when more than one component exists.

shows that the minimal preperiod length is 2 or 3 in each component induced by $D_{P}$, and maximal preperiod lengths are conjectured in [9] (with proofs in some cases). Some additional information about possible preperiod lengths for the $n=T_{k}$ case is given in [6]. However, no general results are known about the distribution of $G E_{P}(n)$ according to preperiod length. Data up to $n=15$ are given in Table 3 .

For GE-compositions, Theorem 3 shows that the minimal preperiod length is 1 in each component induced by $D_{C}$ for $n \geq 4$. There is a general relation between partition and composition preperiod length explored in [9], related to the 'explosion' of the partition $(3,2,1) \vdash 6$ into the six related compositions in $C(6)$ shown in Fig. 6. This allows them to conjecture maximal preperiod lengths, with proofs in some cases. Again, no general results are known about the distribution of $G E_{C}(n)$ according to preperiod length. Data up to $n=10$ are given in Table 4 .

Acknowledgments: We are grateful to Matthew J. Haines for helping with the literature search. Comments from the anonymous referees have helped us improve the article. Also, each author was on sabbatical from his respective institution while undertaking this research. 


\begin{tabular}{|c|c|c|c|c|c|c|c|c|c|c|c|c|c|}
\hline$d \backslash n$ & $\mathbf{3}$ & $\mathbf{4}$ & $\mathbf{5}$ & $\mathbf{6}$ & $\mathbf{7}$ & $\mathbf{8}$ & $\mathbf{9}$ & $\mathbf{1 0}$ & $\mathbf{1 1}$ & $\mathbf{1 2}$ & $\mathbf{1 3}$ & $\mathbf{1 4}$ & $\mathbf{1 5}$ \\
\hline $\mathbf{2}$ & 1 & 1 & 1 & 0 & 1 & $1: 1,0$ & 1 & 0 & 1 & $2: 1,1$ & $2: 1,1$ & 1 & 0 \\
\hline $\mathbf{3}$ & & & 1 & 1 & 3 & $4: 2,2$ & 4 & 1 & 3 & $4: 2,2$ & $6: 4,2$ & 4 & 1 \\
\hline $\mathbf{4}$ & & & & 1 & 1 & $1: 1,0$ & 2 & 3 & 9 & $13: 9,4$ & $13: 7,6$ & 10 & 3 \\
\hline $\mathbf{5}$ & & & & 0 & & $1: 1,0$ & 1 & 1 & 2 & $3: 2,1$ & $5: 3,2$ & 7 & 8 \\
\hline $\mathbf{6}$ & & & & 1 & & & 1 & 1 & 2 & $2: 1,1$ & $3: 2,1$ & 3 & 3 \\
\hline $\mathbf{7}$ & & & & & & & 1 & 1 & 1 & $1: 1,0$ & $2: 1,1$ & 6 & 3 \\
\hline $\mathbf{8}$ & & & & & & & & 2 & 3 & $2: 1,1$ & $5: 5,0$ & 3 & 3 \\
\hline $\mathbf{9}$ & & & & & & & & 1 & & & $1: 1,0$ & 3 & 3 \\
\hline $\mathbf{1 0}$ & & & & & & & & 1 & & & & 1 & 3 \\
\hline $\mathbf{1 1}$ & & & & & & & & 0 & & & & 1 & 2 \\
\hline $\mathbf{1 2}$ & & & & & & & & 3 & & & & 6 & 2 \\
\hline $\mathbf{1 3}$ & & & & & & & & & & & & & 2 \\
\hline $\mathbf{1 4}$ & & & & & & & & & & & & & 2 \\
\hline $\mathbf{1 5}$ & & & & & & & & & & & & & 5 \\
\hline $\mathbf{1 6}$ & & & & & & & & & & & & & 4 \\
\hline $\mathbf{1 7}$ & & & & & & & & & & & & & 3 \\
\hline $\mathbf{1 8}$ & & & & & & & & & & & & & 3 \\
\hline $\mathbf{1 9}$ & & & & & & & & & & & & & 0 \\
\hline $\mathbf{2 0}$ & & & & & & & & & & & & 16 \\
\hline
\end{tabular}

Table 3: The number of GE-partitions of $n$ with preperiod length $d$, broken down by component when more than one component exists.

\section{References}

[1] Ethan Akin, Morton Davis. Bulgarian solitaire, Amer. Math. Monthly 92 (1985) 237-250.

[2] George Andrews. The Theory of Partitions, Cambridge Mathematical Library, Cambridge University Press, 1998.

[3] Elwyn R. Berlekamp, John H. Conway, Richard K. Guy. Winning Ways for your Mathematical Plays, Volume 3, Second Edition, A K Peters, 2004.

[4] Jørgen Brandt. Cycles of partitions, Proc. Amer. Math. Soc. 85 (1982) 483-486.

[5] Arthur Benjamin, Jennifer Quinn. Proofs That Really Count, Dolciana Mathematical Expositions, Mathematics Association of America, 2003.

[6] Hans-J. Bentz. Proof of the Bulgarian solitaire conjectures, Ars Combin. 23 (1987) 151-170.

[7] Gwihen Etienne. Tableaux de Young et solitaire bulgare. J. Combin. Theory Ser. A 58 (1991) 181-197.

[8] Martin Gardner. Bulgarian solitaire and other seemingly endless tasks, Sci. Amer. 249 (1983) 12-21. 


\begin{tabular}{|c|c|c|c|c|c|c|c|c|}
\hline$d \backslash n$ & $\mathbf{3}$ & $\mathbf{4}$ & $\mathbf{5}$ & $\mathbf{6}$ & $\mathbf{7}$ & $\mathbf{8}$ & $\mathbf{9}$ & $\mathbf{1 0}$ \\
\hline $\mathbf{1}$ & 0 & 2 & 3 & 1 & 5 & $8: 5,3$ & 5 & 1 \\
\hline $\mathbf{2}$ & 0 & 0 & 0 & 1 & 7 & $14: 14,0$ & 13 & 5 \\
\hline $\mathbf{3}$ & 1 & 1 & 0 & 0 & 0 & $1: 1,0$ & 3 & 6 \\
\hline $\mathbf{4}$ & & & 4 & 3 & 10 & $11: 5,6$ & 6 & 0 \\
\hline $\mathbf{5}$ & & & 1 & 5 & 20 & $46: 30,16$ & 36 & 18 \\
\hline $\mathbf{6}$ & & & & 1 & 1 & $6: 6,0$ & 61 & 43 \\
\hline $\mathbf{7}$ & & & & 2 & & $7: 7,0$ & 28 & 79 \\
\hline $\mathbf{8}$ & & & & 6 & & $1: 1,0$ & 9 & 8 \\
\hline $\mathbf{9}$ & & & & & & & 10 & 15 \\
\hline $\mathbf{1 0}$ & & & & & & & 30 & 29 \\
\hline $\mathbf{1 1}$ & & & & & & & & 33 \\
\hline $\mathbf{1 2}$ & & & & & & & & 9 \\
\hline $\mathbf{1 3}$ & & & & & & & & 33 \\
\hline $\mathbf{1 4}$ & & & & & & & & 34 \\
\hline $\mathbf{1 5}$ & & & & & & & & 110 \\
\hline
\end{tabular}

Table 4: The number of GE-compositions of $n$ with preperiod length $d$, broken down by component when more than one component exists.

[9] Jerrold R. Griggs, Chih-Chang Ho. The cycling of partitions and compositions under repeated shifts, Adv. in Appl. Math. 21 (1998) 205-227.

[10] Kiyoshi Igusa. Solution of the Bulgarian solitaire conjecture, Math. Magazine 58 (1985) 259-271.

[11] Percy Alexander MacMahon. Memoir on the theory of compositions of numbers, Phil. Trans. 185 (1894), 835 - 901.

[12] Edward F. Moore. Machine models of self-reproduction, Proceedings of Symposia in Applied Mathematics, vol. 14, 17-33, American Mathematical Society, 1962.

[13] Masaru Osima. On the irreducible representations of the symmetric group, Canad. J. Math. 4 (1952) 381-384.

[14] Neil J. A. Sloane. The On-Line Encyclopedia of Integer Sequences, published electronically at http://www.research.att.com/ ${ }^{\sim} \mathrm{nj}$ as/sequences (2005).

[15] Andrew Wuensche, Mike Lesser. The Global Dynamics of Cellular Automata: An Atlas of Basin of Attraction Fields of One-Dimensional Cellular Automata, Santa Fe Institute Studies in the Sciences of Complexity, Addison Wesley Longman, 1992. 